\title{
CORRELAÇÄO ENTRE 0 ESTRESSE DO CUIDADOR E AS CARACTERÍSTICAS CLÍNICAS DO PACIENTE PORTADOR DE DEMÊNCIA
}

\author{
Stella Velasques anderaos Cassis*, Theodora Karnakis, tatiana Alves de Moraes, José antónio Ésper Curiati, Ana Catarina \\ Rodrigues Quadrante, Regina Miksian Magaldi \\ Trabalho realizado pelo Serviço de Geriatria e Centro de Referência em Distúrbios Cognitivos do Hospital das Clínicas da Faculdade de Medicina da \\ Universidade de São Paulo, São Paulo, SP
}

\author{
* Correspondência \\ Hospital das Clínicas \\ Faculdade de Medicina da \\ Universidade de São Paulo \\ Av. Dr. Enéas de Carvalho \\ Aguiar, $155-8^{\circ}$ andar \\ São Paulo - SP \\ Cep 05403-000 \\ stellacassis@yahoo.com.br
}

\begin{abstract}
RESUMO
Oвjetivos. Relacionar estresse dos cuidadores de pacientes dementes com características dos pacientes e cuidadores.

Métodos. Análise de prontuários. Variáveis do paciente: sóciodemográicas, comorbidades, medicamentos (atual e prévio), tempo de história e diagnóstico, tipo da demência e gravidade (cognitiva e funcional), sintomas neuropsiquiátricos e comportamentais. Variáveis do cuidador: sóciodemográficas, vínculo com paciente, tempo de cuidado e se residia com paciente. Sobrecarga avaliada pelo questionário de Zarit.

Resultados. Sessenta e sete pacientes (76,8 anos ( $+6,2), 53,7 \%$ mulheres). Oitenta e dois por cento dos cuidadores são mulheres. Relação direta entre estresse e sintomas comportamentais $(p<0,00 I)$, prejúzo funcional $(p=0,003)$, tempo de história $(p=0,016)$ e de cuidado $(p<0,001)$, diagnóstico prévio $(p=0,016)$ e fato de residir com paciente $(p=0,002)$. Pontuaçốes nos testes cognitivos (Mini Mental e CAMCOG) inversamente proporcionais à sobrecarga $(p=0,005$ e $p=0,023)$. Cuidadores negros apresentaram menor estresse $(p=0,012)$.

Conclusão. Relacionou-se o estresse aos sintomas neuropsiquíatricos e comportamentais, comprometimento funcional e cognitivo, e tempo de história, cuidado e convivência. Encontrada menor sobrecarga em cuidadores negros.

UnITERMOS: Demência. Cuidador. Estresse.
\end{abstract}

\section{INTRODUÇÃO}

Nas últimas décadas, o grande aumento na incidência e prevalência das demências, associado ao envelhecimento populacional, tem direcionado a atenção de pesquisadores de todo o mundo para formas de prevenção e tratamento mais eficazes, visando diminuir o impacto dessa condição nos pacientes e em seus cuidadores ${ }^{1,2}$.

A literatura denota evidências de que há relação entre as características dos pacientes com demência, como alterações comportamentais, e o exame psíquico dos cuidadores². Além disso, características dos próprios cuidadores influenciam o estresse vivenciado pelos mesmos.!

A definição de cuidador é muito discutida. Foi considerado como o membro da família que provia cuidados ao paciente, outrora definido como o indivíduo que residia com o paciente e que atuasse no cuidado por mais de seis meses, ou ainda, como o principal responsável por prover ou coordenar os recursos requeridos pelo paciente. ${ }^{21}$

O conceito de estresse envolve uma resposta biológica/ hormonal ampla, porém na literatura o termo é utilizado como sinônimo de sobrecarga do cuidador. Outros termos utilizados são fadiga e impacto. Referem-se à presença de problemas, dificuldades ou eventos adversos que afetam significativamente a vida de pessoas que são responsáveis pelo paciente.

A sobrecarga emocional vivenciada pelo cuidador pode interferir no cuidado prestado ao paciente, sendo inclusive fator preditor de maior número de hospitalizações entre os pacientes ${ }^{3}$, aumento de institucionalizações' e maior mortalidade entre os cuidadores".

Pesquisas sobre esse tema visam determinar as causas relacionadas ao estresse do cuidador do portador de demência e, dessa forma, auxiliar posteriores abordagens para prevenção e tratamento dessa condiçãa.

O objetivo desta pesquisa foi verificar relações entre o nível de sobrecarga emocional dos cuidadores de pacientes portadores de demência com as características clínicas dos pacientes e variáveis sóciodemográficas dos cuidadores.

$\mathrm{Na}$ literatura mundial, as principais causas de sobrecarga do cuidador do paciente portador de demência são os sintomas comportamentais'. Formulou-se a hipótese de que seriam encontrados dados semelhantes nos resultados deste estudo.

\section{Métodos}

Realizou-se estudo retrospectivo, por meio da análise dos prontuários dos pacientes de 60 anos ou mais, com diagnóstico de demência, segundo os critérios do Diagnostic and Statistical Manual of Mental Disorders (DSM-IV), atendidos de janeiro de 2004 a junho de 2005 no Centro de Referência em Distúrbios Cognitivos do Hospital das Clínicas da Faculdade de Medicina da Universidade de São Paulo (CEREDIC).

O critério de inclusão foi diagnóstico de demência, independente da etiologia (Alzheimer, vascular ou outra). Foram excluídos pacientes ainda em fase de investigação, com depressão, transtorno 
cognitivo leve ou outras causas de déficit cognitivo.

Os prontuários continham, além das informações relacionadas aos pacientes, dados dos seus respectivos cuidadores. $O$ indivíduo que provia cuidados ao paciente na maior parte do tempo era considerado o cuidador.

A pesquisa foi aprovada pela Comissão de Ética para Análise de Projetos de Pesquisa (CAPPesq) da Diretoria Clínica do Hospital das Clínicas e da Faculdade de Medicina da Universidade de São Paulo (protocolo n ${ }^{\circ} 1 / 35 / 05$ ).

As variáveis analisadas em relação ao paciente foram: idade, sexo, raça, escolaridade, ocupação principal, aposentadoria, presença de etilismo, déficit visual e auditivo, número de comorbidades e de medicamentos, tempo de história, gravidade da demência (de acordo com a pontuação em testes de avaliação cognitiva, como o Mini Exame do Estado Menta ${ }^{5,6}$ e o CAMCOG $^{7,8}$ ), grau de comprometimento funcional (pelo Questionário de Atividades Funcionais de Pfeffer $^{9}$ ) e presença de sintomas neuropsiquiátricos e comportamentais (pelo Inventário Neuropsiquiátrico ${ }^{10}$ ), além do tipo de demência e se havia diagnóstico e uso de medicações prévios.

Em relação ao cuidador, verificou-se idade, sexo, escolaridade, ocupação principal, aposentadoria, vínculo com o paciente, tempo de cuidado e se residia na mesma habitação que o paciente. O nível de sobrecarga emocional dos cuidadores baseou-se na pontuação na escala Burden Interview (Zarit \& Zarit, 1987), na versão em português, instrumento padronizado e validado para uso no Brasil. ${ }^{11,12}$

\section{Escalas}

As escalas utilizadas nesse estudo são amplamente utilizadas em pesquisas. O Mini Exame do Estado Mental é um instrumento utilizado para triagem e avaliação da evolução dos distúrbios cognitivos, com pontuação variando de 0 a 30 . As notas de corte dependem do grau de escolaridade, sendo considerados anormais, geralmente, escores menores que 24.

O CAMCOG, uma entrevista estruturada, baseada na seção cognitiva do Cambridge Examination for Mental Disorders of the Elderly, permite a verificação de déficits cognitivos em diferentes domínios (orientação, linguagem, memória, atenção, concentração, praxia, percepção e pensamento abstrato). A pontuação varia de 0 a 107. É descrita nota de corte de 79/80, sendo valores menores indicativos de pior desempenho.

A escala de Pfeffer, composta por 10 itens, evidencia a funcionalidade por meio do grau de independência para realização das atividades instrumentais de vida diária. $O$ escore mínimo é 0 e o máximo é 30. Quanto mais pontos, maior é a dependência do paciente, sendo considerada a presença de prejuízo funcional a partir de um escore de 3.

O Inventário Neuropsiquiátrico é um questionário de 12 itens que possibilita determinar a presença de sintomas neuropsiquiátricos e comportamentais, sua freqüência e intensidade. A pontuação, de 0 a |44, é calculada pela multiplicação da freqüência pela intensidade dos sintomas (delírios, alucinações, agitação psicomotora, depressão, ansiedade, euforia, apatia, desinibição, irritabilidade, comportamento motor aberrante, comportamentos noturnos e alterações alimentares). Quanto maior a pontuação, maior a intensidade e freqüência dos mesmos.

A Burden Interview é uma entrevista de 22 perguntas, que avalia o impacto de doenças mentais e físicas nos cuidadores informais. Varia de 0 a 88. Maior pontuação indica aumento na sobrecarga.

\section{Análise estatística}

Foi realizada análise descritiva das características da amostra. As variáveis categóricas foram analisadas estatisticamente pelo teste $t$ de Student.

Compararam-se as médias da entrevista Zarit entre os cuidadores do sexo masculino e feminino, entre as raças (separadas em branca, parda, negra e amarela), de acordo com a ocupação principal (dividida em atividades domiciliares e outras), entre os aposentados e ativos profissionalmente, conforme o vínculo com o paciente (filho, cônjuge, irmão e outros) e entre cuidadores que habitavam a mesma residência do que o paciente e aqueles que não residiam no mesmo local.

Além disso, as médias do questionário Zarit foram comparadas entre cuidadores de pacientes com presença ou ausência de déficit visual, déficit auditivo, etilismo, diagnóstico prévio, medicação prévia própria para demência, e segundo o tipo de demência (doença de Alzheimer, demência vascular e outras).

Correlação de Pearson foi utilizada para avaliação estatística das variáveis idade, escolaridade, tempo de cuidado e de história, número de comorbidades e de medicações, além das escalas anteriormente referidas: Mini Exame do Estado Mental (MEEM), CAMCOG, Inventário Neuropsiquiátrico (INP) e Pfeffer.

Após essa primeira análise, foi realizada regressão logística para variáveis com $p<0,10$. Para os cálculos, foi utilizado o programa estatístico MINI TAB $\mid 4$.

\section{Resultados}

A amostra foi composta de 67 pacientes. A média de idade foi 76,8 anos (+ 6,2) nos portadores de demência e 58,2 anos (+ 12,5) nos cuidadores. O sexo feminino prevaleceu nos pacientes $(53,7 \%)$, sendo a proporção ainda maior entre cuidadores (82\%). A escolaridade dos pacientes foi em média de 4,9 anos $(+4,5)$ e dos cuidadores, 9,2 anos (+ 5,2).

As médias das pontuações nos testes estão descritas na Tabela I.

A média do número de comorbidades dos pacientes foi 3,16 $(+1,8)$ e do número de medicações diárias que faziam uso foi 3,2 $(+2,3)$. O tempo de cuidado teve média de 2,3 anos $(+1,8)$ e o tempo de história de 2,5 anos $(+1,4)$.

Observou-se relação direta entre pontuações no questionário de Zarit e do Inventário Neuropsiquiátrico $(p<0,00$ I) e da escala de Pfeffer $(p=0,003)$, demonstrando que sintomas neuropsiquiátricos mais intensos e freqüentes, e maior prejuízo funcional para atividades instrumentais da vida diária estão relacionados com maiores níveis de estresse do cuidador.

Pontuações nos testes cognitivos foram inversamente propor- 


\begin{tabular}{lcc}
\hline $\begin{array}{c}\text { Tabela I-Pontuações dos pacientes com demência nos testes de cogniçãoe } \\
\text { funcionalidade, e dos cuidadores no teste de sobrecarga emocional. }\end{array}$ \\
\hline Escalas & Média & Desvio padrão \\
\hline & 16,8 & 5,59 \\
MEEM' $^{\prime}$ & 54,3 & 15,63 \\
INMCOG $^{2}$ & 33,79 & 23,98 \\
NP $^{3}$ & 17,07 & 8,25 \\
Pfeffer & \\
Zariti $^{5}$ & 32,0 & 17,66 \\
\hline
\end{tabular}

IMiniexame do estado mental. Pontuação: 0a30. Valores menores indicam maior comprometimento cognitivo.

2Escala cognitiva do Cambridge Examinationfor Mental Disorders of the Elderly. Pontuação: 0 a 107. Valoresmenoresindicammaior comprometimento cognitivo.

${ }^{3}$ Inventário Neuropsiquiátrico. Valores maiores indicam mais sintomas neuropsiquíátricose comportamentais.

${ }^{4}$ Questionário de Atividades Funcionais de Pfeffer. Pontuação: 0 a 27. Valores maiores indicam maior dependênciafuncional.

${ }^{5}$ Burden Interview. Aplicadaao cuidador. Pontuação:0a 88. Valores maiores indicam maior sobrecarga.

cionais à escala de Zarit (Mini Exame do Estado Mental: $p=0,005$ e CAMCOG: $p=0,023$ ), ou seja, pior desempenho nos testes cognitivos determinou maior sobrecarga. A relação entre a escala de Zarit e as demais escalas estão demonstradas no Gráfico I.

A presença de diagnóstico prévio e o fato do cuidador residir com o paciente também evidenciaram pontuação maior na escala de Zarit ( $p=0,016$ IC95\% -20,29;-2, I7 e p=0,002 IC $95 \%$ $-21,48 ;-5,40$, respectivamente). O mesmo ocorreu em relação ao tempo de história $(p=0,016)$ e ao tempo de cuidado $(p<0,00$ I). Isso significa aumento no impacto emocional proporcional ao tempo de evolução da doença e ao tempo de convívio com o paciente.

As demais variáveis (incluindo escolaridade) não demonstraram correlação estatística com níveis de sobrecarga emocional do cuidador, com exceção do nível de sobrecarga emocional entre os cuidadores da raça negra, que foi menor em relação às demais etnias $(p=0,012 \quad$ IC95\% $\quad 1,76 ; 13,33)$.

As variáveis com $p<0,10$ na análise univariada foram consideradas como possíveis variáveis preditoras. Essas variáveis foram: raça, o fato do cuidador residir com o paciente, presença de diagnóstico prévio, uso anterior de medicação específica para demência, idade e escolaridade do cuidador, idade do paciente, tempo de cuidado CAMCOG, MEEM, Pfeffer, INP e tempo de história.

O método de seleção de variáveis forward selecionou as seguintes variáveis: raça, o fato do cuidador residir com o paciente, presença de diagnóstico e medicamento prévio, tempo de cuidado, CAMCOG, MEEM, Pfeffer, INP e tempo de história.

O modelo ajustado foi:

Zarit $=8,85+(8,01 \times 0$ fato do cuidador residir com 0 paciente $)+(0,233 \times$ tempo de cuidado $)+(0,319 \times$ INP $)$

$\mathrm{Na}$ equação de regressão acima, 8,85 é uma constante. $\mathrm{O}$ fato do cuidador residir com o paciente é um preditor que significa 0 quando não ocorre e I quando ocorre, ou seja, o fato de habitar a mesma residência aumenta a pontuação na escala Zarit, significando maior estresse, em 8,01 pontos. O tempo de cuidado, dado

\section{Figura I- Relação entre as escalas MEEM', CAMCOG ${ }^{2}$, INP $^{3}$, Pfeffer $^{4}$ e Zarit $^{5}$}

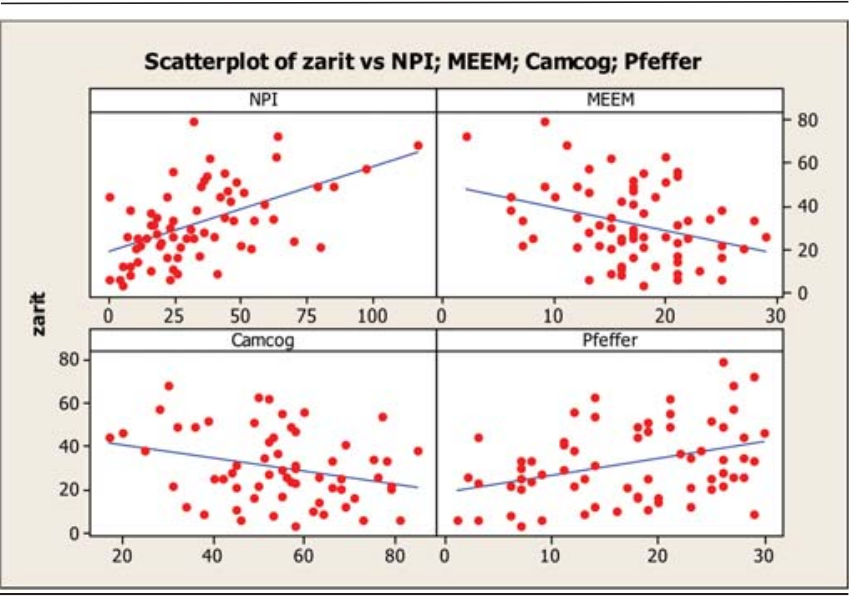

'Miniexame do estado mental. Pontuação: 0a30. Valores menores indicam maior comprometimento cognitivo.

${ }^{2}$ Escala cognitiva do Cambridge Examinationfor Mental Disorders ofthe Elderly. Pontuação: 0 a 107. Valoresmenoresindicammaior comprometimento cognitivo.

${ }^{3}$ Inventário Neuropsiquiátrico. Valores maiores indicam mais sintomas neuro-psiquíátricose comportamentais.

${ }^{4}$ Questionário de Atividades Funcionais de Pfeffer. Pontuação: 0 a 27. Valores maiores indicam maior dependênciafuncional.

${ }^{5}$ Zarit Burden Interview. Aplicada ao cuidador. Pontuação:0a 88. Valores maiores indicam maior sobrecarga.

pelo número de meses, aumenta o Zarit 0,233 vezes; a pontuação do NPI, 0,319 vezes. O ajuste do modelo foi avaliado por meio de uma análise de resíduos.

\section{Discussão}

Neste estudo verificou-se a relação entre as variáveis sociodemográficas do cuidador e as características do paciente portador de demência com o estresse do cuidador. Diversos estudos abordaram este tema, em sua maioria com pesquisas direcionadas aos distúrbios comportamentais dos pacientes $1,2,13,14,15,1617,18,19$. Houve também trabalhos destacando a raça do cuidador $^{20}$ e o vínculo do mesmo com o paciente ${ }^{19}$.

Algumas publicações analisaram as características sociodemográficas dos cuidadores. Torti et al. (2004) encontraram, em revisão multinacional, achados de que cuidadores do sexo feminino carregavam uma sobrecarga emocional particularmente mais penosa nas culturas, principalmente nas sociedades asiáticas.

Em nossa amostra, não houve diferença em relação ao sexo, tanto do paciente como do cuidador, como preditor da sobrecarga emocional do cuidador, assim como não havia alteração em relação às raças, exceto a negra. Salientamos que na amostra deste estudo os cuidadores da raça amarela eram poucos (dois).

Em concordância com nossos resultados, Toth-Cohen (2004) relatou que cuidadores negros de pessoas com doença de Alzheimer e outras demências relacionadas possuíam níveis de estresse significantemente menores do que os de cuidadores brancos. O autor referiu que razões para esta diferença são obscuras e que não podem ser atribuídas somente à raça. 
Não verificamos alterações influenciadas pelo vínculo entre paciente e cuidador, nem como pela idade de ambos. Diferentemente, Croog et al. (200I) analisaram o estresse entre cuidadores de cônjuges de pacientes com doença de Alzheimer e demonstraram que entre esposas cuidadoras e maridos cuidadores jovens (64 anos de idade ou menos) os problemas comportamentais do paciente foram significantemente associados negativamente com 0 total de pontos em uma medida de bem-estar.

Nesse mesmo estudo, verificou-se ainda que maridos cuidadores apresentaram maior ansiedade em relação às esposas cuidadoras. Garrido e Almeida (1999) observaram em revisão que cuidadores do sexo feminino apresentaram maiores índices de sobrecarga e depressão.

A escolaridade, as ocupações dos cuidadores e dos pacientes e o fato de estarem aposentados ou não também não evidenciaram influência no estresse. De acordo com a literatura, esses dados foram inconsistentes nas publicações anteriores.'

As comorbidades, número de medicações em uso, déficits visual e auditivo e a presença de etilismo ou depressão no paciente também não alteraram a sobrecarga emocional do cuidador, assim como o tipo de demência.

Em relação às variáveis que estavam relacionadas ao tempo que o cuidador convivia com o paciente, ou seja, se residia com o paciente, o tempo de cuidado, o tempo de história e se havia diagnóstico prévio, encontramos maior sobrecarga quanto maior o tempo. O tempo de cuidado foi semelhante ao tempo de história, indicando que os cuidadores assumiram essa função desde o início dos sintomas.

Destacaram-se também às correlações com as escalas de gravidade de demência e de funcionalidade do paciente. Esses dados são divergentes na literatura.

Garrido e Almeida (1999) citam as divergências, como a ausência de influência do estado cognitivo do paciente no impacto do cuidador e o decréscimo dos níveis de sobrecarga com o passar do tempo em alguns trabalhos. Danhauer et al. (2004) também descrevem este processo adaptativo e a falta de aumento sistemático no estresse, apesar da maior gravidade dos sintomas de demência.

Considerando a história natural das síndromes demenciais, o tempo médio de 2,5 anos de cuidado, encontrado na amostra estudada, pode ser avaliado como um período curto para o desenvolvimento desse processo adaptativo.

Já Torti et al. (2004) descreveram que cuidadores que despendiam maior tempo com os pacientes apresentavam maior depressão e ansiedade, considerando o fato de residirem com o paciente. Relatam também que quanto maior a gravidade da demência, maiores os sintomas somáticos, ansiedade e depressão, e menor o bem-estar dos cônjuges cuidadores. Esse efeito foi observado nos aspectos comportamentais, não cognitivos, da gravidade da doença.

Todos os estudos concordavam que o impacto ou sobrecarga sofridos na vida do cuidador, assim como o desenvolvimento de sintomas depressivos, tinham relação direta com a presença de transtornos psiquiátricos apresentados pelos pacientes.
Porém, Garrido e Almeida (1999) ressalvam a heterogeneidade das alterações do comportamento estudadas e da própria falta de definição para o conceito de cuidador e de estresse.

$\mathrm{Na}$ amostra estudada, assim como em todos os estudos da revisão bibliográfica que realizamos, o maior preditor de estresse evidenciado encontra-se nos sintomas comportamentais, relação mais estudada $1,2,13,14,15,16,17,18,19$. Este trabalho corrobora esta clara evidência: entre os três preditores de estresse está a maior pontuação no Inventário Neuropsiquiátrico, em conjunto com o fato do cuidador residir com o paciente e o tempo de cuidado.

\section{Conclusão}

$\mathrm{Na}$ amostra analisada, maiores níveis de estresse do cuidador relacionaram-se à presença de sintomas neuropsiquiátricos e comportamentais do paciente, ao grau de comprometimento funcional, à gravidade do déficit cognitivo, ao tempo de história de sintomas de demência, ao tempo de cuidado, ao fato do cuidador residir com o paciente e de haver diagnóstico prévio. Menor sobrecarga foi encontrada em cuidadores da raça negra.

Intervenções futuras para reduzir o estresse do cuidador devem considerar essas características e as divergências discutidas para identificar pacientes e cuidadores sob maior risco e desenvolver estratégias que diminuam esse impacto.

\section{Agradecimentos}

À Adriana Nunes Machado e à Carmem Saldiva, pela colaboração na análise estatística. À Daniela Velasques Arce, pela colaboração na tradução e revisão.

Conflito de interesse: não há.

\section{SUMMARY}

\section{Correlation between burden on Caregiver and Clinical CHARACTERISTICS OF PATIENTS WITH DEMENTIA}

OBJECTVE. To verify the correlation between caregiver burden in dementia and characteristics of patients and caregivers.

Methods. Analysis of medical records. Patient data: sociodemographic characteristics, comorbidities, medications (previous and current), onset of diagnosis and symptoms, type of dementia and severity (cognitive impairment and functional abilities), behavioral disorders. Caregiver data: socio-demographic characteristics, kinship, duration of caregiving and co-residency with the patient. Caregiver burden assessed by the Zarit interview.

RESULTS. Sixty seven patients (76.8 years $(+6.2), 53.7 \%$ women) and $82 \%$ female caregivers were surveyed. Correlation between burden and behavior disorders $(p<0.001)$, dependencies $(p=0.003)$, onset of symptoms $(p=0.016)$ and of caregiving $(p<0.001)$, previous diagnosis $(p=0.016)$ and coresidency $(p=0.002)$ was studied. Cognitive test scores (Mini Mental and CAMCOG) were inversely proportional to distress $(p=0.005$ and $p=0.023)$. Black caregivers demonstrated lower levels of stress $(p=0.012)$. 
Conclusion. Burden was associated with behavioral disorders, dependencies, cognitive impairment, and onset of symptoms, caregiving and co-residency. Black caregivers demonstrated lower levels of stress. [Rev Assoc Med Bras 2007; 53(6): 497-50I]

KeY wORDS: Dementia. Caregiver. Burden.

\section{REFERÊNCIAS}

I. Torti FM, Gwyther LP, Reed SD, Friedman JY, Schulman KA. A multinational review of recent trends and reports in dementia caregiver burden. Alzheimer Dis Assoc Disord. 2004;18:99-109.

2. Gaugler JE, Zarit SH, Pearlin LI. The onset of dementia caregiving and its longitudinal implications. Psychol Aging. 2003;18:17|-80.

3. Balardy L. Predictive factors of emergency hospitalisation in Alzheimer's patients: results of one-year follow-up in the REAL.FR Cohort. J Nutr Health Aging. 2005;9:1/2-6.

4. Patterson TL, Grant I. Interventions for caregiving in dementia: physical outcomes. Curr Opin Psy chiatr. 2003;16:629-33.

5. Folstein MF, Folstein SE, McHugh PR. Mini Mental State: A practical method for grading the cognitive state of patients for the clinician. J Psychiatr Res. 1975;12:189-98.

6. Brucki SMD, Nitrini R, Caramelli P, Bertolucci PHF, Okamoto $I H$. Sugestões para o uso do mini-exame do estado mental no Brasil. Arq Neuropsiquiatr. 2003;61:777-81.

7. Roth M, Tym BK, Mountjoy CQ, Huppert FA, Hendrie H, Verma S, et al. CAMDEX: a standardized instrument for the diagnosis of mental disorders in the elderly with special reference to the early detection of dementia. Br J Psychiatry. 1986;149:698-709.

8. Bottino C, Stoppe Jr A, Scalco AZ, Ferreira RCR, Hotatian SC, Scalco MZ. Validade e confiabilidade da versão brasileira do CAMDEX. Arq Neuropsiquiatr. 2001;59(Supl 3):20.

9. Pfeffer RI, Kurosaki TT, Harrah CH Jr, Chance JM, Filos S. Measurement of functional activities in older adults in the community. J Gerontol. 1982;37:323-9.

1 0. Cummings JL, Mega M, Gray K, Rosenberg-Thompson S, Carusi DA, Gornbein J, et al. The Neuropsychiatric Inventory: comprehensive assessment of psychopathology in dementia. Neurology. | 994:44:2308-14

I I. Scazufca M. Brazilian version of the Burden Interview scale for the assessment of burden of care in carers of people with mental illnesses. Rev Bras Psiquiatr. 2002;24:12-7.

12. Taub A. Dementia caregiver burden: reliability of the Brazilian version of the Zarit caregiver burden interview. Cad Saude Publica. 2004;20:372-6.

13. Schulz R, Belle SH, Czaja SJ, Mc Ginnis KA, Stevens A, Zhang S. Longterm care placement of dementia patients and caregiver health and wellbeing. JAMA. 2004:292:961-7.

14. Covinsky KE. Dementia, prognosis and the needs of patients and caregivers. Ann Intern Med. 2004; I40: 573-5.

15. Danhauer SC, Beckett LA, McCann JJ, Gilley DW, Bienias JL, Evans DA. Do behavioral disturbances in persons with Alzheimer's disease predict caregiver depression over time?. Psychol Aging. 2004;19:198-202.

16. Gitlin LN, Burgio LD, Mahoney D, et al. Effect of multicomponent interventions on caregiver burden and depression: the REACH multisite initiative at 6-month follow-up. Psychol Aging. 2003;|8:36|-74.

17. Pinquart M, Sörensen S. Differences between caregivers and noncaregivers in psychological health and physical health: a metaanalysis. Psychol Aging. 2003; 18:250-67.

18. Clark PC, King KB. Comparison of family caregivers: stroke survivors vs. persons with Alzheimer's disease. J Gerontol Nurs.2003;29:4553.

19. Croog SH, Sudilovsky A, Burleson JA, Baume RM. Vulnerability of husband and wife caregivers of Alzheimer disease patients to caregiving stressors. Alzheimer Dis Assoc Disord. 2001;15:201-10.

20. Toth-Cohen S. Factors of influencing appraisal of upset in black caregivers of persons with Alzheimer disease and related dementias. Alzheimer Dis Assoc Disord. 2004;18:247-55.

21. Garrido R, Almeida OP. Distúrbios de comportamento em pacientes com demência. Arq Neuropsiquiatr. 1999;57:427-34.

Artigo recebido: 24/01/06

Aceito para publicação: 25/09/07 